This item was submitted to Loughborough's Research Repository by the author.

Items in Figshare are protected by copyright, with all rights reserved, unless otherwise indicated.

\title{
Analysis of wood surface waviness with a two-image photometric stereo method
}

PLEASE CITE THE PUBLISHED VERSION

PUBLISHER

Professional Engineering Publishing / @ IMECHE

VERSION

VoR (Version of Record)

LICENCE

CC BY-NC-ND 4.0

\section{REPOSITORY RECORD}

Jackson, Michael R., D. Yang, and Robert M. Parkin. 2019. "Analysis of Wood Surface Waviness with a Twoimage Photometric Stereo Method". figshare. https://hdl.handle.net/2134/4694. 
This item was submitted to Loughborough's Institutional Repository (https://dspace.lboro.ac.uk/) by the author and is made available under the following Creative Commons Licence conditions.

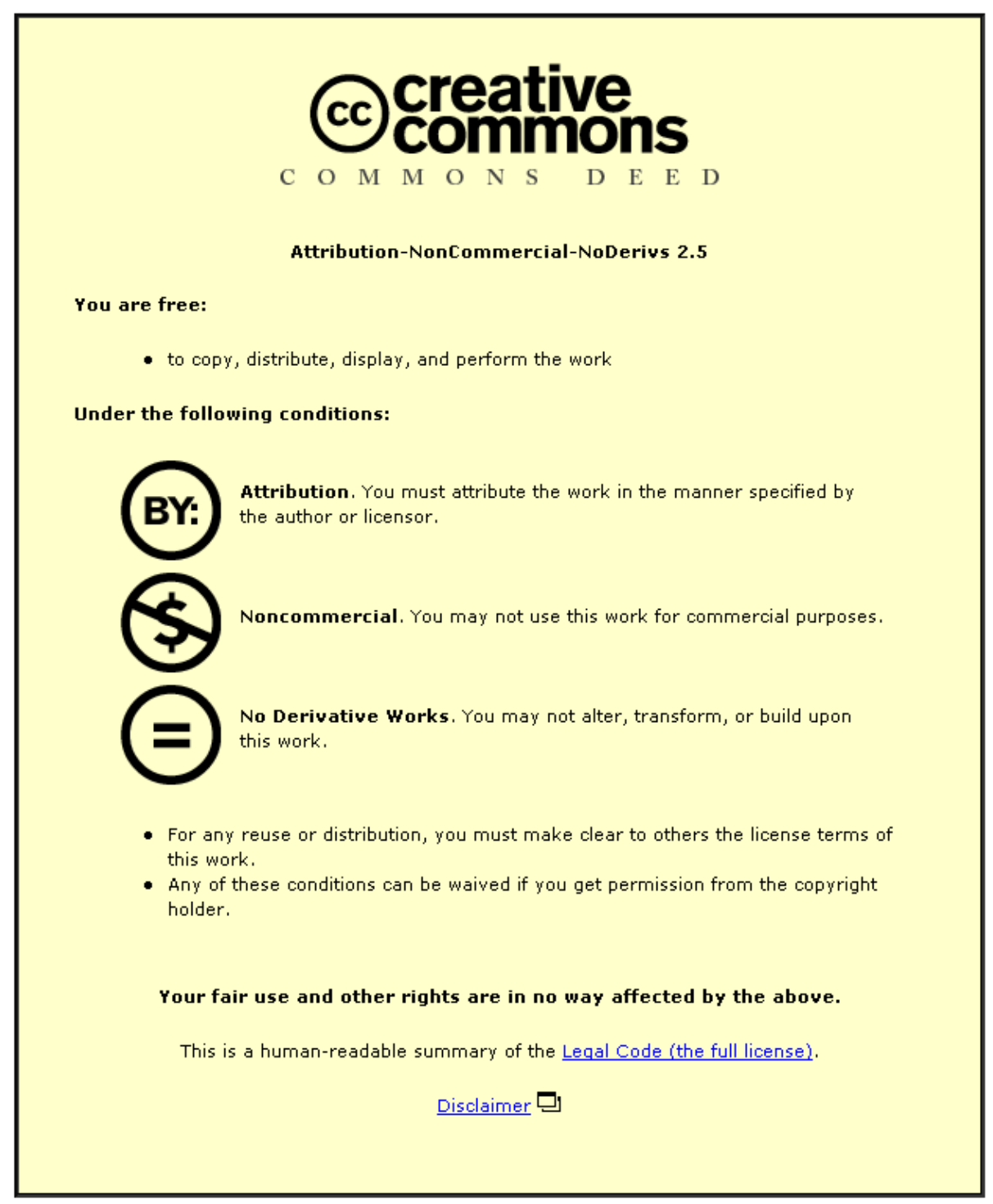

For the full text of this licence, please go to: http://creativecommons.org/licenses/by-nc-nd/2.5/ 


\title{
Analysis of wood surface waviness with a two-image photometric stereo method
}

M Jackson*, D Yang, and R Parkin

Mechatronics Research Centre, Loughborough University, Loughborough, UK

The manuscript was received on 13 January 2007 and was accepted after revision for publication on 31 July 2007.

DOI: 10.1243/09596518JSCE405

\begin{abstract}
This paper describes a two-image photometric stereo (PS) method for analysis of surface waviness on planed wood products. This method is based on an assumption that the wood surface is a Lambertian surface. In addition, owing to the fact that surface waviness on planed wood is mainly composed of cutter marks, which fluctuate only in one dimension, three-dimensional surface topography can reasonably be represented by a two-dimensional surface profile. Accordingly, a surface waviness profile can be extracted from two images of the surface under specifically arranged illumination. Measurement results from the two-image PS method are compared with those from a laser profilometer. Comparison indicates a high correlation between the two methods.
\end{abstract}

Keywords: wood surface, waviness, Lambertian surface, photometric stereo

\section{INTRODUCTION}

In some sectors of the woodworking industry, such as furniture manufacturing, surface quality determines the market value. As a result, it is highly desirable to have a capability of assessing final finish of machined wood surfaces. Among a number of wood surface quality characteristics, surface waviness is an important aspect. Surface waviness on wood, medium-wavelength surface height variations formed in the planing processes, primarily comprises cutter marks. Figure 1 illustrates the formation of cutter marks in the planing process.

In order to assess waviness on machined wood surfaces, various methods have been employed. Stylus tracing is traditionally used, but the usage of this technique on wood has some problems, which are discussed by Jackson $[\mathbf{1}, 2]$. Sandak et al. and Lemaster et al. employed optical sensors to assess wood surface quality [3-7]. Essentially, however, the optical sensors Sandak and Lemaster used are optical versions of stylus tracers with light as styli. All kinds of stylus tracing device have a common problem with

\footnotetext{
* Corresponding author: Wolfson School of Mechanical Engineering, University of Loughborough, Loughborough, Leicestershire LE11 3TU, UK. email: m.r.jackson@lboro.ac.uk
}

wood surface measurement: each scanning of the stylus across the surface can only produce a single surface profile, which may very well misrepresent the three-dimensional topographic surface because of the anisotropic nature of wood. This problem can certainly be solved by averaging multiple surface profiles, but it is usually time consuming to trace a large number of surface profiles, especially in an online manner.

Yang et al. measured wood surface waviness using the light sectioning method [8]. According to the method, a narrow light stripe is projected on to the measured surface at a very small angle, and the light stripe projection on the surface is termed a light section. Since there are all kinds of surface feature, including waviness, on the surface, the light section will consequently reveal a surface profile, from which a waviness profile can be extracted. Compared with the stylus tracing, the light sectioning method is much more efficient because a single light section embodies hundreds of sampling points. However, similar to stylus tracing, a surface profile obtained from a light section may still misrepresent the three-dimensional topographic surface.

Hoffmeister and Grubler $[\mathbf{9}, \mathbf{1 0}]$ took another approach to assess wood surface waviness, which is based on analysis of intensity variations across an 


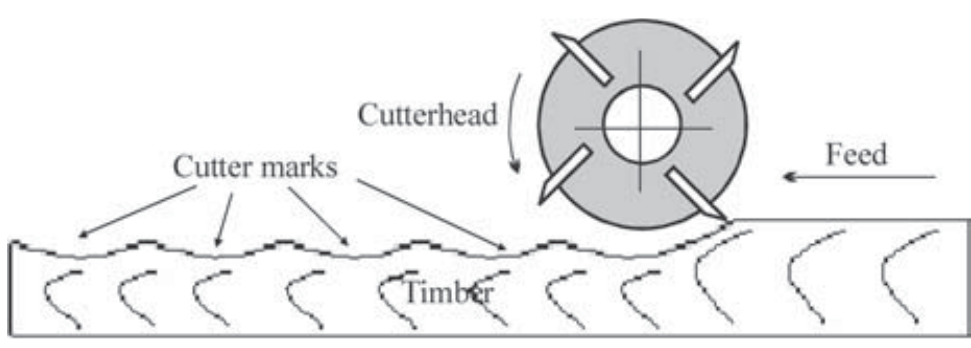

Fig. 1 The formation of cutter marks on wood surfaces in the planing and moulding processes

obliquely illuminated surface. Hoffmeister's method works in terms of measuring waviness widths. However, it is unable to reveal waviness height information.

Surface waviness is a subset of surface topography. Therefore, surface waviness measurement is also a kind of surface topography measurement. Horn introduced a method known as 'shape from shading' (SFS) to determine surface topography from a single image [11]. Woodham extracted surface topography from multiple images with different illumination conditions [12]. Methods based on the detection of multiple images are commonly referred to as 'photometric stereo' (PS) methods.

In this paper, a two-image photometric stereo method for measuring wood surface waviness is proposed. First, some theories are introduced, and then experiments are described, followed by results and conclusions. This work is part of a longer-term research project aimed at active control of the timber machining process.

\section{THEORY}

The PS method is always based on some kind of surface reflection model. Usually, the Lambertian model is employed because of its effectiveness and simplicity. This section first introduces the Lambertian model, and then describes the two-image PS method.

\subsection{Lambertian model}

When light strikes the surface of an opaque material, part of the radiation energy will be reflected. Reflection can occur in one of the three manners illustrated in Fig. 2. When the material is a conductor and has a perfectly mirror-like surface, the reflection is solely directional and termed specular reflection. When the material is a dielectric and has a smooth surface such as painted wood surfaces, the reflection will have a diffuse dome with a specular lobe in one direction, as illustrated in Fig. 2(b). When the material is a dielectric exhibiting surface texture, then the reflection will behave as shown in Fig. 2(c), with a diffuse dome. This type of reflection (c) is known as Lambertian reflection, and the surface as a Lambertian surface.

Reflection from a dielectric surface such as machined wood consists of specular reflection and diffuse reflection. Diffuse reflection must be separated from specular reflection before Lambert's law can be applied to extract surface features. The separation of diffuse reflection from specular reflection has been carried out on planed wood surfaces using a dichromatic reflection model [13]. The light reflected from these surfaces is observed as being largely diffuse in nature, indicating that planed wood surfaces exhibit Lambertian properties.

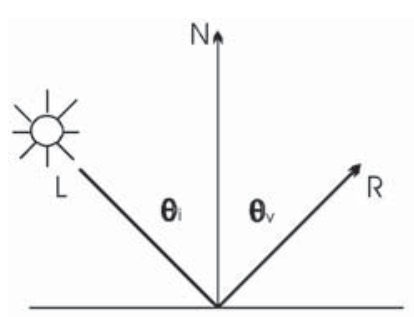

(a)

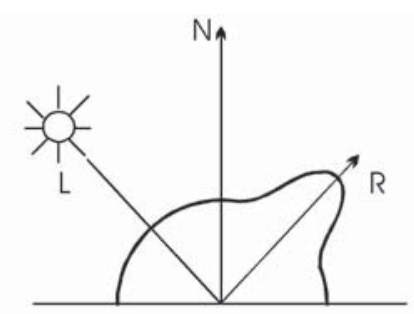

(b)

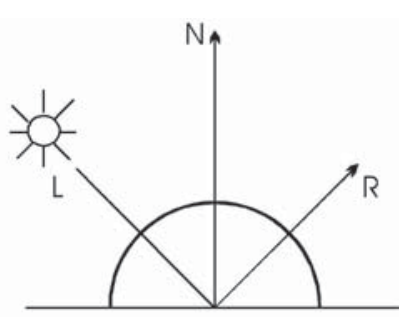

(c)

Fig. 2 Surface reflection models: (a) mirror-like specular, (b) specular + diffuse, (c) perfectly diffuse 
Reflection from a Lambertian surface is schematically illustrated in Fig. 3, where:

the $X-Y$ plane is coincident with the surface; the $Z$ axis is the global normal to the surface; $(x, y)$ is a point on the surface;

$N$ is the local normal to the point $(x, y)$;

$\boldsymbol{L}$ is the vector pointing from the point $(x, y)$ to the light source;

$\sigma$ is the angle between $\boldsymbol{L}$ and the $Z$ axis, in the range $0-90^{\circ}$;

$\tau$ is the angle between $L$ and the $X$ axis measured in the $X Y$ plane, in the range $0-360^{\circ}$;

$\gamma$ is the angle between $L$ and $N$ in the range $0-90^{\circ}$.

According to Lambert's law [13], reflection at a surface point $(x, y)$ is determined by

$$
\begin{aligned}
i(x, y)= & i_{0} \rho(x, y) \frac{-p(x, y) \cos \tau \sin \sigma}{\sqrt{p^{2}(x, y)+q^{2}(x, y)+1}} \\
& -\frac{q(x, y) \sin \tau \sin \sigma+\cos \sigma}{\sqrt{p^{2}(x, y)+q^{2}(x, y)+1}}
\end{aligned}
$$

where

$i(x, y)$ is the intensity reflected at the point $(x, y)$; $i_{0}$ is the incident intensity;

$\rho(x, y)$ is the surface albedo, a coefficient that represents the proportion of light reflected from the point $(x, y)$ with respect to the incident light;

$p$ and $q$ are partial derivatives of the surface function $s(x, y)$, i.e.

$$
p(x, y)=\frac{\partial s(x, y)}{\partial x} \quad \text { and } \quad q(x, y)=\frac{\partial s(x, y)}{\partial y}
$$

Since the objective of this research is to measure cutter mark profiles on planed wood surfaces, the generalized Lambertian model can be simplified. Compared with surface roughness, i.e. randomly distributed short-wavelength surface height variations, surface waviness on planed wood has a directional pattern, as illustrated in Fig. 4. This allows a twodimensional two-image PS method to be developed. This approach requires two light sources (one for each image) to allow extraction of surface features.

\subsection{The two-image PS method}

In principle, the wood surface has no height variations along the $Y$ axis in Figs 3 and 4, i.e. $q(x, y) \equiv 0$ in equation (1). In addition, if the two light sources are located opposite to each other and both along the waviness direction, i.e. $\tau_{1}=180^{\circ}$ and $\tau_{2}=0^{\circ}$, with the $X$ axis coincident with the waviness direction, then the reflection model in Fig. 3 is simplified to that in Fig. 5. Collimated light sources LS1 and LS2 are identical in intensity, and symmetric with respect to the camera. The annotations in Fig. 5 are as follows.

$S$ is the surface normal, coincident with the $Z$ axis and the optical axis of the camera;

$x$ is a point on the surface (surface profile, to be precise);

$n(x)$ is the normal to the point $x$;

$\boldsymbol{L 1}$ is the vector pointing from the point $x$ to LS1;

$\boldsymbol{L} \mathbf{2}$ is the vector pointing from the point $x$ to LS2;

$\sigma_{1}$ and $\sigma_{2}$ are the angles between the surface normal $S$ and $\boldsymbol{L} \mathbf{1}$, and $S$ and between $\boldsymbol{L} \mathbf{2}$ respectively; since these two angles are equal, $\sigma$ is used;

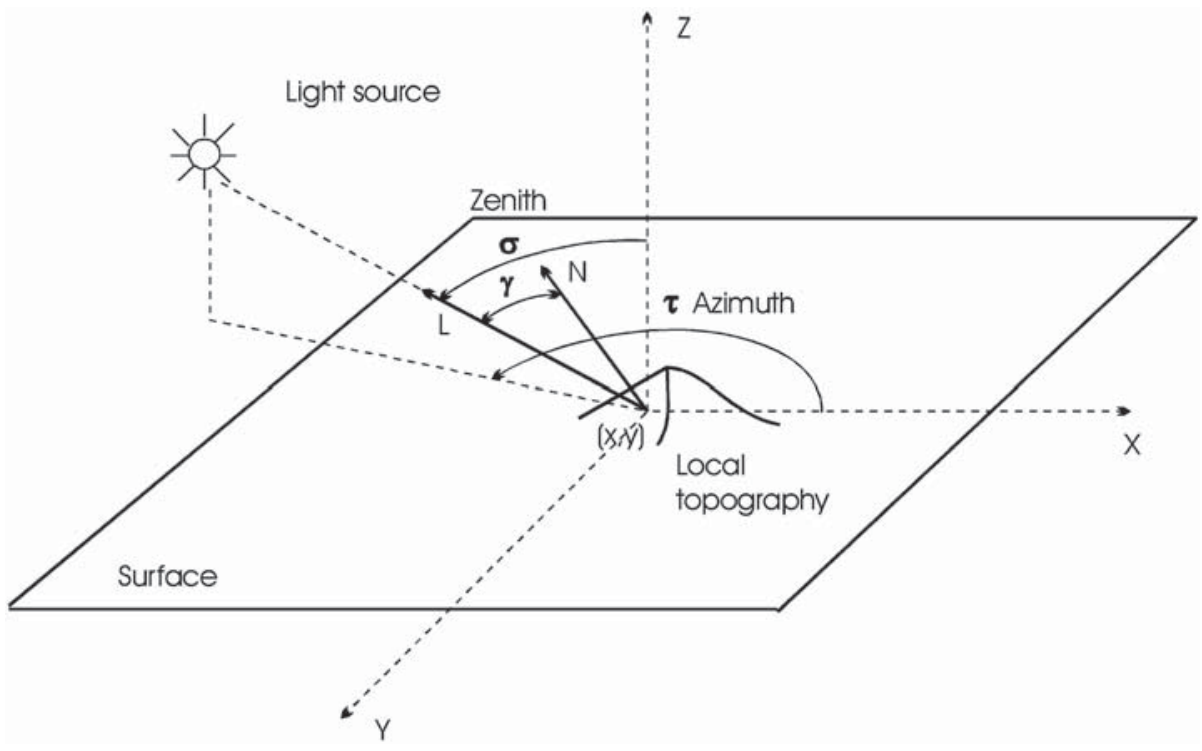

Fig. 3 Schematic of Lambertian reflection 


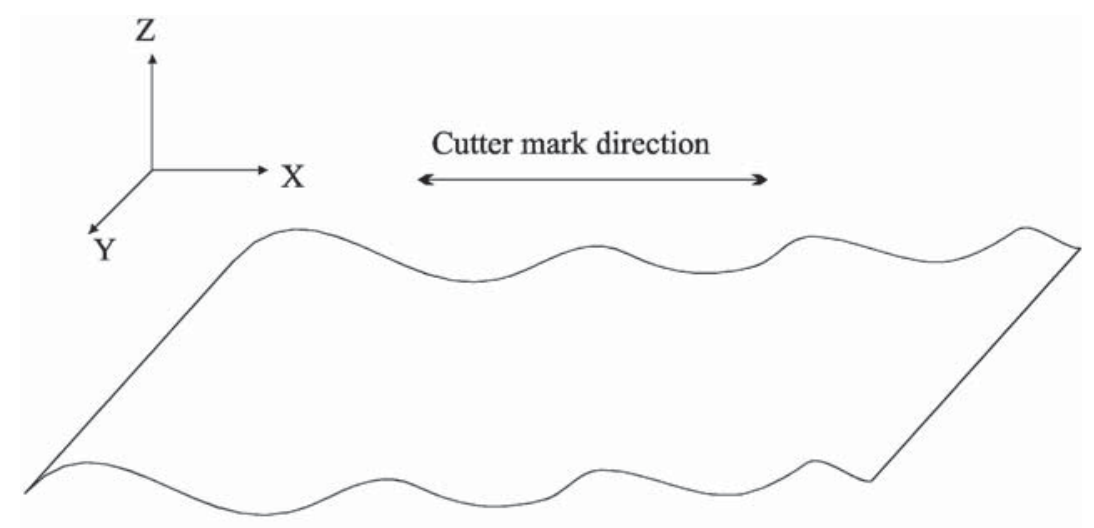

3D surface with cutter marks

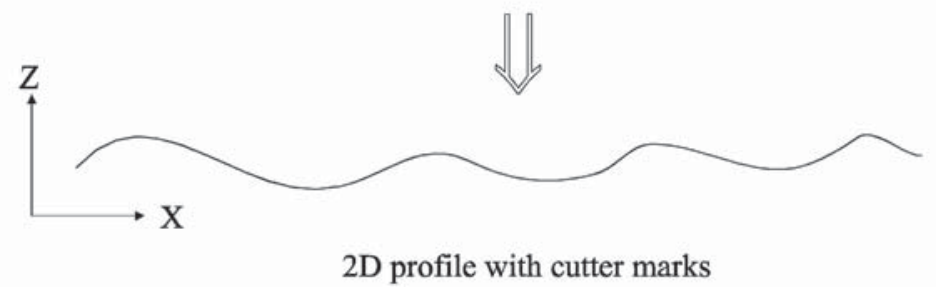

Fig. 4 Two-dimensional profile representing surface waviness

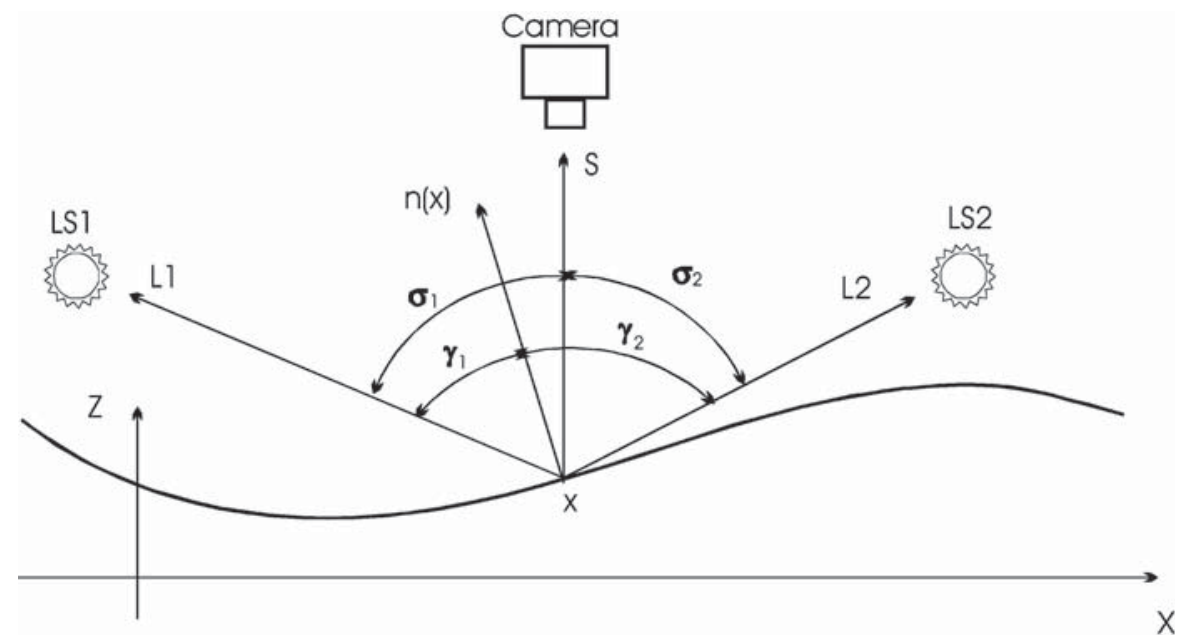

Fig. 5 Schematic of the two-image photometric stereo method

$\gamma_{1}$ is the angle between the normal $n(x)$ and $\boldsymbol{L 1}$; $\gamma_{2}$ is the angle between the normal $n(x)$ and $\boldsymbol{L} 2$.

Substitute $q(x, y)=0, \tau_{1}=180^{\circ}$, and $\tau_{2}=0^{\circ}$ into equation (1)

$$
\begin{aligned}
& i_{1}(x)=i_{01} \rho(x) \frac{p(x) \sin \sigma+\cos \sigma}{\sqrt{p^{2}(x)+1}} \\
& i_{2}(x)=i_{02} \rho(x) \frac{-p(x) \sin \sigma+\cos \sigma}{\sqrt{p^{2}(x)+1}}
\end{aligned}
$$

where $i_{01}=i_{02}$, as LS1 and LS2 are identical in intensity.
Dividing equation (2) by equation (3) gives

$$
p(x)=\frac{i_{1}(x)-i_{2}(x)}{i_{1}(x)+i_{2}(x)} \frac{1}{\tan \sigma}
$$

Accordingly, the first derivative of the surface function $p(x)$ can be derived from two images of the surface. Similar to the simplification of the threedimensional surface to a two-dimensional profile, the images need to be converted to intensity profiles. This can be implemented by using an algorithm introduced in references [9] and [10]. The algorithm, 
referred to as 'averaging column by column', is illustrated in Fig. 6.

Symbol $g_{i j}$ is the greyscale value (intensity value) of the pixel at the $i$ th row and $j$ th column of the image matrix. Symbol $c(j)$ is the summation of greyscale values of all the pixels in the $j$ th column as shown by

$$
c(j)=\frac{\sum_{m} g_{i j}}{m}
$$

The intensity profiles $c(j)$ for each of the two surface images are substituted for $i_{1}(x)$ and $i_{2}(x)$ in equation (4) to calculate $p(x)$, the first derivative of the surface $s(x)$. In order to extract the surface function $s(x)$ from $p(x)$ it is necessary to apply appropriate integration techniques.

\subsection{Integration of $p(x)$}

After substitution of the $c(j)$ intensity profiles into equation (4), the first derivative (slope) of the surface $p(x)$ results, as shown by

$$
p(x)=\frac{\mathrm{d} s}{\mathrm{~d} x}
$$

Equation (6) needs to be integrated. Two possible integration techniques could be: (a) local approaches and (b) global approaches [14]. Local approaches start integration with an initial depth and propagate depth values according to a local approximation rule. Some local integration techniques estimate the depth of a point by considering the gradients of two adjacent points, while some consider more points for accuracy. Several global integration techniques

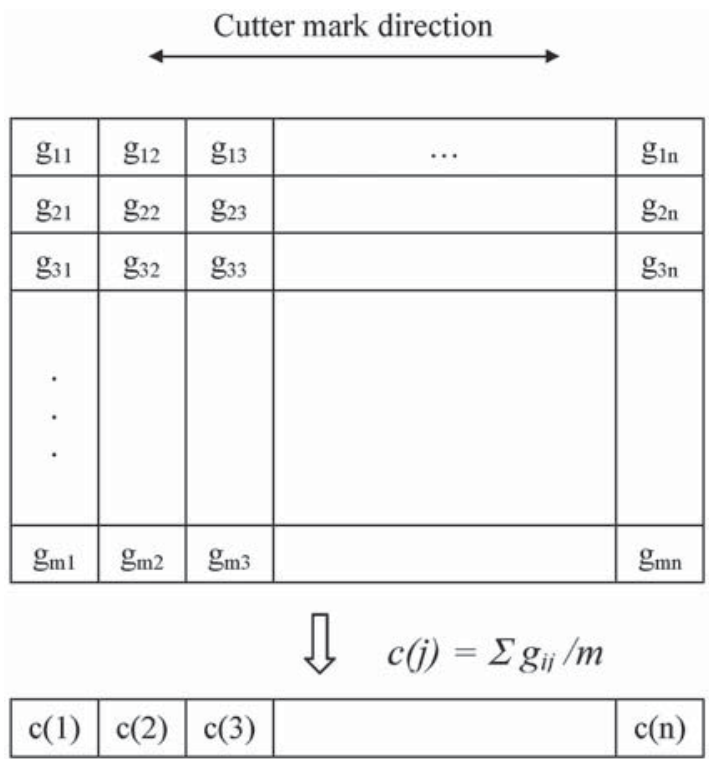

Fig. 6 The algorithm of averaging column by column have been developed. Frequency integration is a global integration technique, which is considered less complex and most relevant.

A frequency integration algorithm is adapted from reference $[\mathbf{1 5}]$. The Fourier transformation of equation (6) gives

$$
P(u)=2 \pi i u S(u)
$$

Then

$$
S(u)=\frac{P(u)}{2 \pi i u}
$$

Finally

$$
s(x)=F^{-1}(S(u))
$$

A local integration algorithm for the twodimensional surface is also developed. This approach is a propagation integration technique. The procedure for the algorithm is as follows.

1. Suppose $s(1)=0$.

2. $s(i+1)=s(i)+p(i), i \geqslant 1$.

3. Repeat step 2 until the whole surface is recovered.

An experiment shows that frequency integration and propagation integration give very similar results.

\section{EXPERIMENT}

A PS test rig was constructed on the basis of the principles outlined in section 2 . The test rig has the capability to image moving timber samples up to speeds of $2 \mathrm{~m} / \mathrm{s}$. Experiments reported here are for stationary timber samples.

\subsection{Experimental set-up}

The experimental set-up is illustrated in Fig. 7. Collimated light sources LS1 and LS2 are located on opposite sides of the surface, and are symmetric to the camera. Figure 8 shows the physical arrangement of the test rig. The overall size is $800 \mathrm{~mm} \times 800 \mathrm{~mm} \times$ $1200 \mathrm{~mm}$ high. The system is composed of a variablespeed transport conveyor, a Prosilica 1/2" CCD (charge coupled device) $640 \times 480$ pixel Firewire camera, two high-intensity LED (light-emitting device) light sources, an incremental angular encoder, a proximity sensor, and a PIC18F458 microcontroller board. The two light sources (LS1 and LS2) are identical in terms of intensity, the angle relative to the conveyor surface, and the distance from the camera. The wood sample is placed underneath the camera and the camera acquires an image with LS1 on; then the camera acquires another image with LS2 on. 


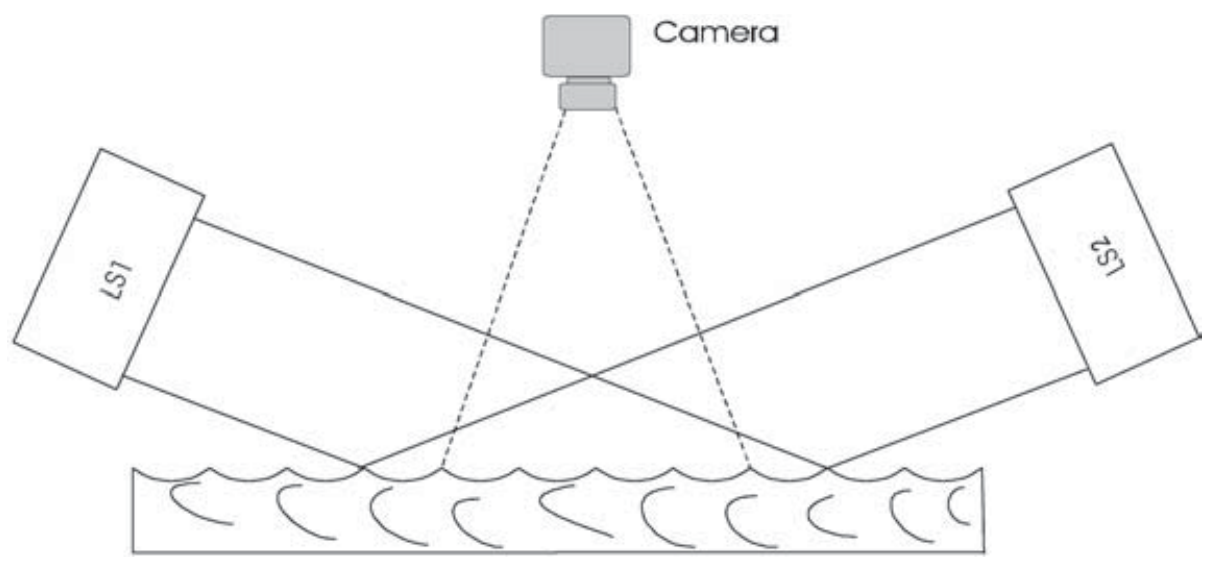

Fig. 7 Experimental set-up

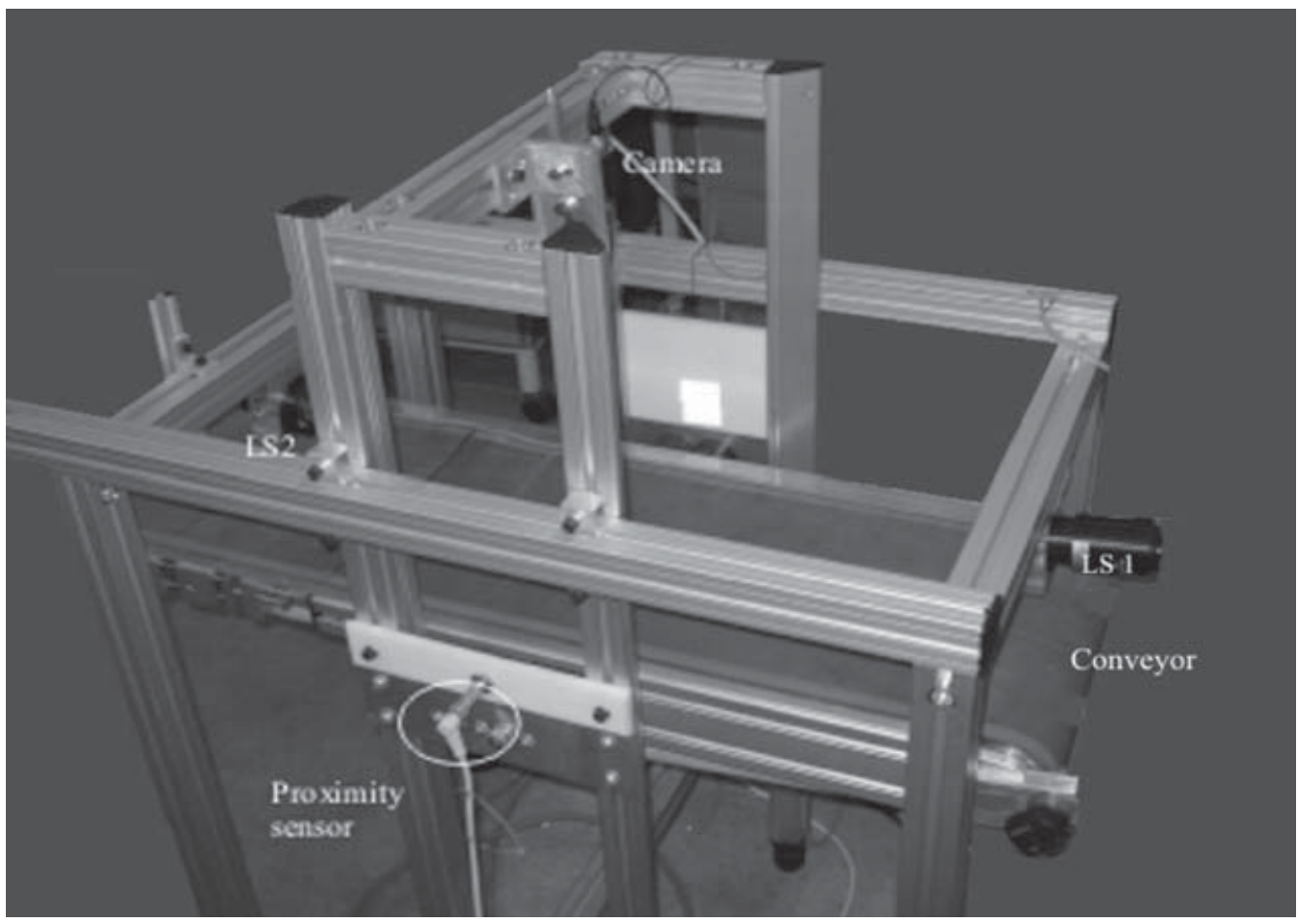

Fig. 8 Photometric stereo test rig

Beech, ramin, and oak wood samples were measured using the test rig. Each sample is $180 \mathrm{~mm} \times$ $60 \mathrm{~mm} \times 20 \mathrm{~mm}$ in size. The wood species were chosen for the degree of challenge in terms of wood surface waviness measurement. Cutter marks on beech show up well visually because of the hard and relatively consistent grain texture. Ramin is a soft hardwood with consistent grain, but cutter marks are less obvious than on beech. Oak has a hard consistency, but cutter marks are sometimes obscured by the grain texture. Each type of wood has a machined waviness of $2.5,2$, and $1.5 \mathrm{~mm}$ in width respectively. The samples were prepared with a cutter of $60 \mathrm{~mm}$ in cutting radius. The camera was mounted perpendicular to the timber surface, with a field of view (FOV) of $40 \times 32 \mathrm{~mm}$ for samples with a waviness width of $2.5 \mathrm{~mm}$ and an FOV of $20 \times 16 \mathrm{~mm}$ for samples with waviness widths of 2 and $1.5 \mathrm{~mm}$.

\subsection{Measurement reference}

In order to assess the results from the two-image PS method, a laser profilometer was used as a reference. The laser profilometer is based on an autofocusing technique. This approach was used in a previous investigation on light sectioning [8]. Detailed information about the autofocusing technique can be found in reference [16]. The vertical resolution 
of the profilometer is $20 \mathrm{~nm}$, and the horizontal sampling interval was set to $25 \mu \mathrm{m}$ for samples with waviness widths of 1.5 and $2 \mathrm{~mm}$, and $50 \mu \mathrm{m}$ for samples with a waviness width of $2.5 \mathrm{~mm}$. The profilometer is a very slow instrument and not suitable for real-time surface measurement. The instrument does, however, provide a baseline comparison with newly developed methods for wood surface measurement. However, even the profilometer cannot reproduce a consistent surface form owing to the small focus spot size being affected by the wood grain surface texture. For this reason the results from comparisons between the profilometer and the PS instrument have been shown visually as surface traces and also as correlation values. Producing tables of differences between the two instruments can result in large values $(\sim 2-5 \mu \mathrm{m})$ at certain points of the trace, given the unpredictable effects of timber grain texture.

\subsection{Results}

Figure 9 shows typical images of timber surfaces obtained with the PS test rig. The surfaces exhibit subtle differences when illuminated from the righthand side (LS1) and the left-hand side (LS2). Image processing using the theory and method outlined in section 2, using the Matlab Image Processing toolbox, allows the surface waviness to be revealed. Surface profiles obtained with the two-image PS test rig and those measured with the laser profilometer are compared in terms of correlation coefficients. The comparison is shown in Table 1. From the table, it can be seen that measurements from the two methods are highly correlated.

Three pairs of surface profiles are plotted in Fig. 10 and also indicate the agreement between the twoimage PS method and the laser profilometer. Some differences in traced profiles are evident $(\sim 3 \mu \mathrm{m})$, but, since there is no instrument that measures timber surface quality to a true baseline reference, it cannot be stated whether these differences result from the laser profilometer, the PS instrument, or both. What is clear is that both methods produce similar results.

\section{DISCUSSION}

Compared with the conventional stylus tracing and optical stylus tracing techniques, the two-image PS method has the advantage of being able to extract information from an area rather than from only a few scanned traces. This advantage of the two-image PS method is also applicable to the light sectioning

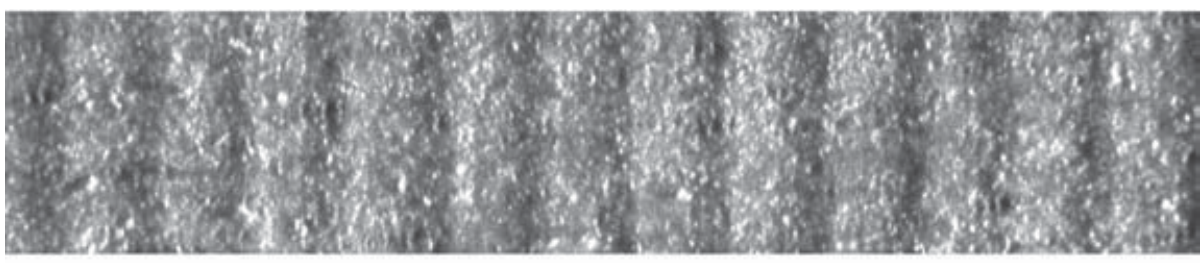

(a) Illuminated from right hand side (LS1)

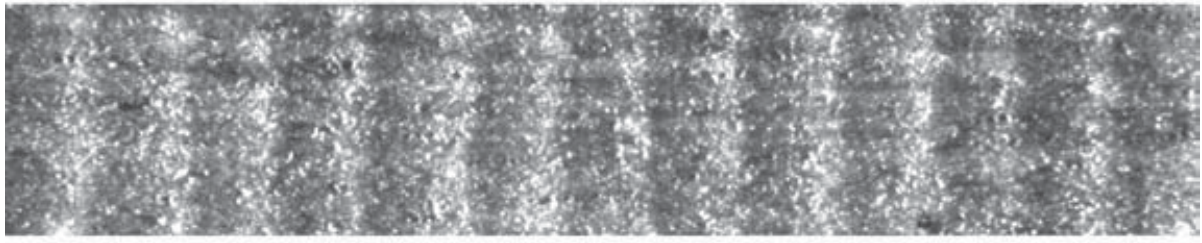

(b) Illuminated from left hand side (LS2)

Fig. 9 Typical images of timber surfaces obtained with the PS test rig

Table 1 Correlation coefficients for surface profiles measured with the PS method and the laser profilometer

\begin{tabular}{|c|c|c|c|c|c|c|c|c|}
\hline \multicolumn{3}{|c|}{ Beech } & \multicolumn{3}{|c|}{ Ramin } & \multicolumn{3}{|c|}{ Oak } \\
\hline $1.5 \mathrm{~mm}$ & $2 \mathrm{~mm}$ & $2.5 \mathrm{~mm}$ & $1.5 \mathrm{~mm}$ & $2 \mathrm{~mm}$ & $2.5 \mathrm{~mm}$ & $1.5 \mathrm{~mm}$ & $2 \mathrm{~mm}$ & $2.5 \mathrm{~mm}$ \\
\hline 0.82 & 0.86 & 0.94 & 0.88 & 0.94 & 0.80 & 0.83 & 0.88 & 0.83 \\
\hline
\end{tabular}



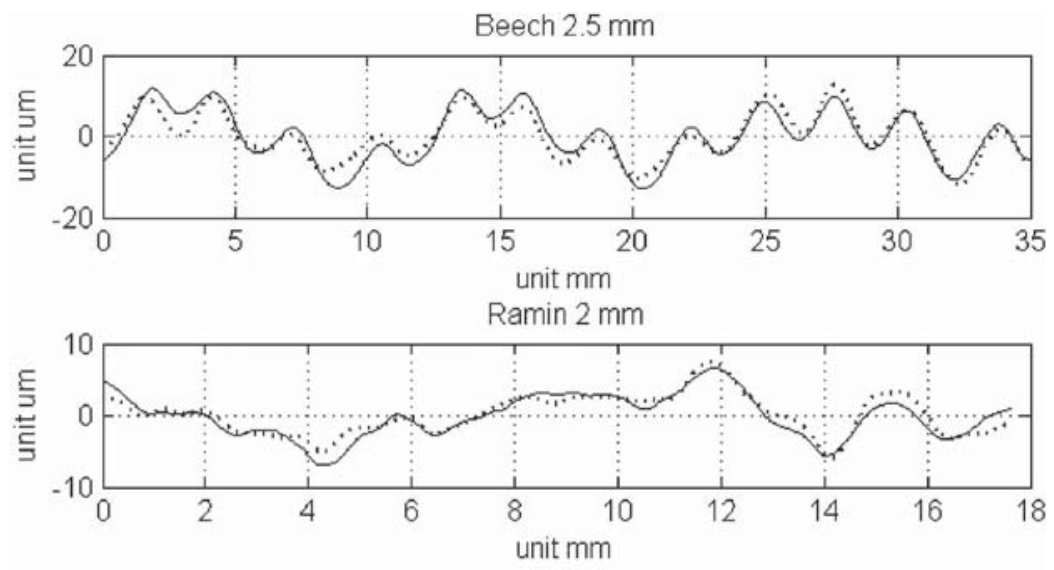

Oak $1.5 \mathrm{~mm}$

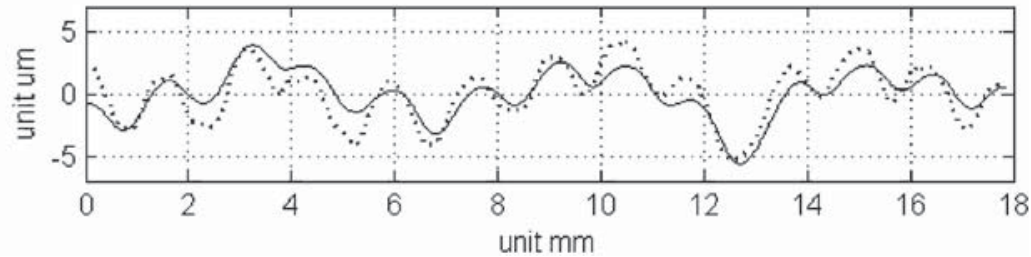

Fig. 10 Surface waviness profiles measured with the PS method (solid lines) compared with profiles measured with a laser profilometer (dotted lines)

method [8], which is similar to stylus tracing in the sense that measurement is only based on individual profiles obtained from light sections. From this point of view, the two-image PS method is more reliable in terms of describing surface waviness on wood over an area. Also, the two-image PS method is more efficient since scanning of the surface is no longer needed.

Compared with the method developed by Hoffmeister et al., the two-image PS method has a capability of producing surface profiles, from which both waviness heights and widths can be obtained.

\section{CONCLUSIONS AND FUTURE WORK}

The two-image PS method proposed in this paper for measuring surface waviness on planed wood has a solid theoretical foundation, and has proven viable in the initial experimental work on static wood samples. Compared with the other methods, the two-image PS method has advantages of being able to measure widths and heights of waviness over an area and being more efficient and reliable.

Future work will focus on further verification of the two-image PS method by measuring more wood species with smaller waviness (width $<1.5 \mathrm{~mm}$ ) and measuring moving samples at speeds up to $2 \mathrm{~m} / \mathrm{s}$ using the test rig shown in Fig. 8.

\section{ACKNOWLEDGEMENT}

The work reported here was supported by the EPSRC Innovative Manufacturing and Construction Research Centre (IMCRC) at Loughborough University.

\section{REFERENCES}

1 Jackson, M. R. Some effects of machine characteristics on the surface quality of planed and spindle moulded wooden products. PhD Thesis, Leicester Polytechnic, 1986.

2 Jackson, M. R., Parkin, R. M., and Brown, N. Waves on wood. Proc. Instn Mech. Engrs, Part B: J. Engineering Manufacture, 2002, 216(B4), 475-497.

3 Sandak, J., Tanaka, C., and Ohtani, T. Evaluation of surface smoothness by laser displacement sensor. I: effect of wood species. J. Wood Sci., 2003, 49(4), 305-311.

4 Sandak, J., Tanaka, C., and Ohtani, T. Evaluation of surface smoothness by a laser displacement sensor. II: comparison of lateral effect photodiode and multielement array. J. Wood Sci., 2004, 50(1), 22-27.

5 Lemaster, R. L. and Beall, F. C. The use of an optical profilometer to measure surface roughness in medium density fiberboard. Forest Products J., 1996, 46(11/12), 73-78.

6 Lemaster, R. L. and Taylor, J. B. High speed surface assessment of wood and wood-based composites. In The 14th International Wood Machining Seminar, Epinal, France, 1999. 
7 Lemaster, R. L., Lu, L., and Jackson, S. The use of process monitoring techniques on a CNC wood router. Part 1: sensor selection. Forest Products J., 2000, 50(7/8), 31-38.

8 Yang, D., Jackson, M., and Parkin, R. Inspection of wood surface waviness defects using the light sectioning method. Proc. IMechE, Part I: J. Systems and Control Engineering, 2006, 220(I7), 617-626.

9 Hoffmeister, H. W. and Grubler, T. InProzessmessung der Messerschlagweite gehobelter Massivholzoberflächen. HOB Die Holzbearbeitung, 1999, 5, 200-204.

10 Hoffmeister, H. W. and Grubler, T. Objektive Bestimmung von Jointintervallen durch Messung der Messerschlagweite. HOB Die Holzbearbeitung, 2000, 9.

11 Horn, B. K. P. Understanding image intensities. Artif. Intell., 1977, 8, 201-231.
12 Woodham, R. J. Photometric method for determining surface orientation from multiple images. Opt. Engng, 1980, 19(1), 139-144.

13 Maristany, A. G., Lebow, P. K., Brunner, C. C., Butler, D. A., and Funck, J. W. Classifying woodsurface features using dichromatic reflection. In Proceedings of the SPIE - The International Society for Optical Engineering, 1836 (1992), pp. 56-64.

14 Gullon, C. Height recovery of rough surfaces from intensity images. $\mathrm{PhD}$ Thesis, Herriot-Watt University, 2003.

15 Frankot, R. T. and Chellappa, R. A method for enforcing integrability in shape from shading algorithms. IEEE Trans. Pattern Analysis and Machine Intell., 1988, 10(4), 439-451.

16 Griffiths, B. Manufacturing surface technology: surface integrity and functional performance, 2001 (Penton, London). 